\title{
Walk 2 Win: Towards Designing a Mobile Game for Elderly's Social Engagement
}

\author{
Omar Mubin \\ Dept. of Industrial Design, \\ Eindhoven University of \\ Technology, Eindhoven, \\ the Netherlands \\ o.mubin@tue.nl
}

\author{
Suleman Shahid \\ Dept. of Communication and \\ Information Sciences, \\ University of Tilburg, \\ the Netherlands \\ s.shahid@uvt.nl
}

\author{
Abdullah Al Mahmud \\ Dept. of Industrial Design, \\ Eindhoven University of \\ Technology, Eindhoven, \\ the Netherlands \\ a.al-mahmud@tue.nl
}

\begin{abstract}
In this paper we describe a user-centered approach to designing and evaluating a socially interactive mobile game for the elderly. The objective of the game is to encourage the elderly to socially engage amongst themselves and to make the time that they spend in the community center more enjoyable. The design of the game, known as Walk 2 Win was done for and with the elderly. Two levels of the game (easy, difficult) across two modes (single, multi player) were evaluated with the elderly in a community center over two sessions. Our results show that senior citizens are keen to play simple games having uncomplicated rules as long as they can customize the game according to their whims. Their preference lies in playing games that are enhancements of existing games that they usually play. It was found that the elderly expressed a strong preference and interest to build a team, with for example their grand children. Additionally it was revealed that games for the elderly should incorporate everyone, regardless of gender or their role in the game. The methodological lessons that we came across throughout the design process are also presented.
\end{abstract}

\section{Categories and Subject Descriptors}

H.5.2 [Information Interfaces and Presentation]: User Interfaces - user centered design, prototyping, evaluation/methodology

\section{General Terms}

Design, Experimentation, Human Factors.

\section{Keywords}

Ethnography, focus group, fun, game, elderly, user-centered design.

\section{INTRODUCTION}

Current society is confronted by a new challenge due to demographical changes that is the population of older people in the world is increasing. On the other hand older people are becoming more isolated due to family breakdowns [5]. A large part of elderly live at home and there is not enough means for them to alleviate their isolation and loneliness. The rapid

(c) The Author 2008

Published by the British Computer Society growth of technology has clearly made the digital divide gape even more widely and has alienated the elderly. There is an urgent requirement to involve the elderly in activities that can be their source of fun and entertainment and at the same time improve their physical and psychological well being. One example of such an activity is gaming, particularly games that require physical play in social settings. With the passing of age, the elderly are threatened by various physical and psychological problems, which are usually not addressed by conventional games, resulting in the fact that the elderly miss out on this important aspect of game play. More so, there is hardly any game out there which primarily addresses the social need of the elderly to bond with their peers and/or family members.

There have been some games developed for the elderly for instance, digital games that are played on computer [6]. These games are either video games used for therapy [2], [3], [4], or to improve social and educational skill. Persuasive games have been designed to keep people fit [7] e.g. Nintendo Wii. It has been established that traditional digital games have merits nevertheless they barely meet the needs of the growing elderly community. One of the main reasons that behind the computer games create social isolation is due to the lack of face to face communication with opponents or co-players. On the one hand, most of the existing computer games are tailored for adolescents and not seniors and on the other computing game technology is almost always deployed in a non-social setting, which leads to frustration and social isolation due to an over dependency on the digital world. The utilization of computer games for purposes such as entertainment, socialization and stimulating memory can put a positive impact on the elderly if games can be redesigned by taking into account the real needs of the user group [8]. Since most of the elderly people have limited social contacts, there is a dire need for designing a game that can motivate them to be socially engaged in their everyday life. In addition to overcoming technological obstacles and for motivating the elderly to play games, the focus should be shifted such that new games are designed that are a part of the real environment and part of their everyday activities. We particularly focus on how we can design a game for the elderly which may aid them to be more active with their cohorts while socializing in the community center.

Our endeavor will contribute to understanding the need of designing a new simple, enjoyable and socially entertaining game for the elderly. We consider our design case study as an initial investigation into user-centered game design for senior citizens, in order to unearth several principles that can be added as game elements, such as social interaction, easy and customizable game play, etc.

In the following section we describe the data gathering process, followed by the game implementation process. Finally the game evaluation and the design implications are discussed. 


\section{REQUIREMENTS CAPTURING}

Our user study session comprised of three phases and our research was concentrated on a local community center for the elderly. A community center is a club like gathering where people usually visit for socialization and to carry out activities on the spot for instance drinking coffee and having a chat.

Firstly, we ran an ethnographic study in the community center for the elderly. Secondly, we interviewed the care givers of the same community center. Lastly, we organized a focus group session with the elderly. Our main findings are summarized below.

Games Played: The most popular games played by the elderly include: billiards (played by men only), card games (e.g., bank, poker), and guessing and memory games (see Figure 1). The elderly also like to play quiz (games that relate to the use of knowledge) and memory games (e.g. word construction, kind of scrabble). The participants claimed to have limited technological capability with respect to advanced games. They at times play games on their cell phone, for e.g. Snake, but those games are at not very technologically advanced. Another game activity that they carry out is drawing and sketching (mostly women). Some people also bring their own games along with them. The caregiver said, "Time flies when they play games that they actually want to play".

Game features - Likes and Dislikes: It was revealed that the choices the elderly make in playing games were influenced by the games shown on various television shows. We also learnt that interestingly, most of the elderly enjoyed playing games with their grandchildren, as they love bonding with their grandchildren and the children also assist them in understanding the rules. They knew about computer and console games for e.g. FIFA and Mario Bros as these games were played by their grandchildren. They thought that even console games can be interesting as long as they are close to real life.

Finally, the participants talked about what they do not appreciate about some of the games they know. The elderly within our user group claimed that playing Scrabble using a game board and alphabets was not interesting to them. However, when they played Scrabble with simplified rules and technological aid it was more exciting. They mentioned that it was very difficult to find how to play certain games as they forget the instructions and rules easily, for e.g. if a particular game has too many controls or options. The elderly were not very confident about their game playing skills with regards to fast and swift games. Consequently, their grandchildren would not wish to be in the same team as them in such games. The elderly also pointed out that they do not like playing animated games as it makes them immature.

Social Interaction Trends: Passive Game play was observed and recorded. It was found to be an important aspect of elderly game play. Non players were found to be watching others play and commenting on the game. It was also noted that most of the elderly carry out their activities in groups. The caregiver also stressed the importance of the social communication amongst the members of the club. The elderly usually visit the community center as a purpose to interact with each other. They have some planned activities e.g. fitness, game playing rounds and an arts session. But they also have unplanned sessions where a few people just drop in randomly and start doing a social activity spontaneously.
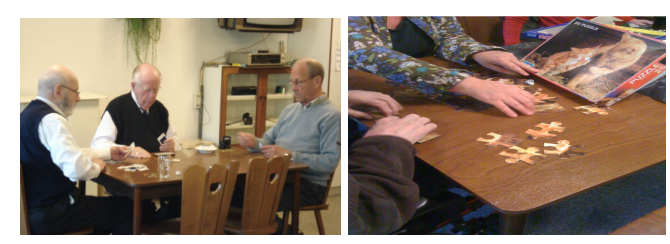

Figure 1. Snapshots from the Data gathering phase.

\section{GAME DESIGN PROCESS}

From the results of our user study we identified three key design principles which were to be incorporated into our final game design:

- To design a game which encourages senior citizens to be more active in participation (both active and passive involvement).

- Which endorses them to interact with each other during game play.

- Which has simple rules yet provides fun.

The elderly have been playing various standard games for a long time but at times there is nothing original in the games that they play. Occasionally, this leads to a sense of boredom for the players. Within the design team we realized that it was, firstly imperative to supplement existing conventional games by integrating features such as technological advancements and secondly any game for the elderly should be built on the basis of flexible rules.

To move towards our final design we conducted several brainstorming sessions within the research team. We decided that we would focus on memory, physical activity and customizability as the key elements of our new game. Before implementing our game we brought forward an initial paper mock up and tested it with four elderly users in the same local community center. The elderly gave us valuable suggestions with regards to the design choices in the game. For instance, some of the game features we had designed were disapproved by the elderly as childish game elements. We finalized our game concept and in the next section we describe the rules of our game: Walk 2 Win (W2W).

\subsection{Walk 2 Win}

$\mathrm{W} 2 \mathrm{~W}$ is a mobile memory game for the elderly, consisting of two game modes (individual player or playing as a team) and two game levels (easy and difficult). At one time four players, each having a mobile device, either in teams or individually can play the game. When the game starts, players see a screen asking them to choose how they want to play the game: individually or in teams (see Figure 2). The system then asks them to enter their name/team name and creates a unique account for them and establishes a connection with the server. After this, players are asked to choose whether they start with the easy or difficult level (see Figure 2). Once the level is selected, all the players play in the same level (a consensus is developed before starting the game).

In the first round (easy level), players see six cards, placed face down ( 2 rows with 3 cards) on the screen of their mobile device. Behind each card lies a unique object (famous personality etc). In total there are 3 objects and the goal of the player is to complete all 3 pairings (see Figure 3 ). Each pair is also assigned with a sound of the particular object, so that when 
a card is revealed, the corresponding sound is played by the PDA.

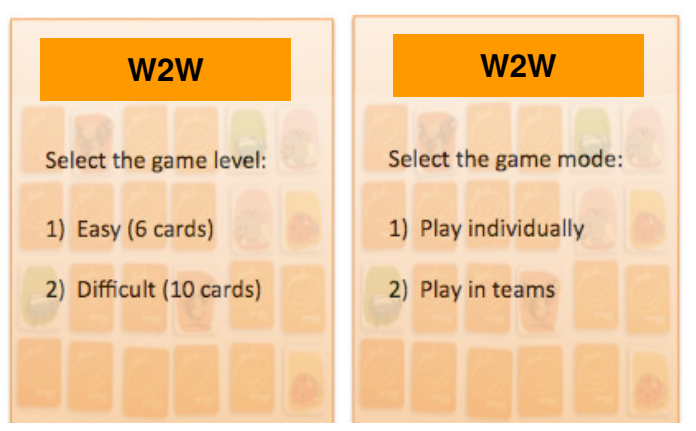

Figure 2. Menu screens

There are six ventilation holes marked as wireless hot spots in the game room. The goal of a team is to find the three pairs of the cards associated with the hot spots. To overturn the card a player approaches towards the hot spot and tries to activate it by moving his/her mobile device over the hot spot. If successful, a sound is generated and a corresponding card is revealed. Now the player (or his/her team-mate) has to search for the other spot in the room, which should disclose an identical picture as shown on the previously displayed card. If the player is able to discover two similar cards consecutively then both cards are unlocked and a hurrah sound is played. If the player is unable to find the exact same card in the next turn, then a boo sound is played and the currently visible card is turned upside down. Now the player has to start searching again. The same rules are applied for searching all pair of cards. The level is finished when a team has found all three pairs and all pairs are unlocked on both mobile devices. Now if players wish they can move to the next level and start playing there. The next level is the difficult stage, where players are faced with 5 pair of cards.

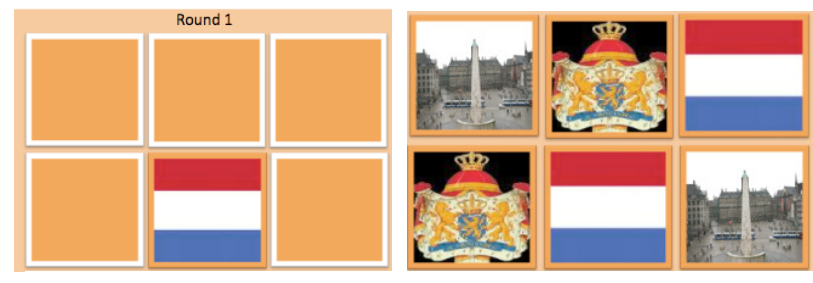

Figure 3. Card pairs

If the players are playing individually then there is a variation in the game rule. When one player has found all the cards, he is considered first but the game continues and other players are allowed to search the pairs and compete for second and third positions. This game finishes when the first three players have found the pairs.

\subsection{Prototype Implementation}

The game interface was developed using DHTML (integrated sound and images). There were two interfaces of the game. One was the admin interface from where game is controlled (adding/deleting cards, setting sounds) and other is the user interface, which a player sees on his/her mobile device. The user interface is a dynamic HTML page that refreshes itself after every two seconds, reflecting any changes in the game environment.

\section{GAME EVALUATION}

The game was evaluated to scrutinize whether the elderly comprehend the game rules, whether they perceive the technological aspects of the game, and finally whether they perceive any fun in the game.

\subsection{Method}

The game was evaluated in two sessions (individual and team mode) with 8 voluntary participants (5 males and 3 females), average age: 71 (from 67 to 78 ) in the same local community center. All were healthy and could walk without any assistance. Only three of them had computer experience and all of them played card games.

The evaluation session was 2 hours long with 2 breaks. An A3 size print out was also posted on the wall, which briefly described the game rules. Before the actual game was played, a small introductory pilot session was carried out to ensure that the rules were fully understood. Each player was given a mobile device and was allowed exploration time with the device (see Figure 4). We did not have permission to record the session.

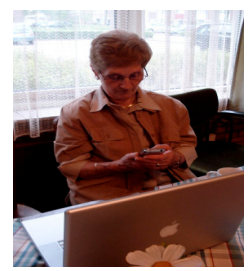

Figure 4. Free Exploration with the Mobile Device.

\subsection{Game Setup}

From the four PDA's, two were HTC cruise with touch screen functionality (the elderly preferred touch over stylus based interaction) and two of them were the iPhone. An ad-hoc WIFI network was built and Mac book Pro's wireless card was used as a wireless access point. Each PDA was connected to the Mac book pro, which was actually hosting the game (see Figure 5). The revealing and hiding of the cards in the case of proximity to a particular hotspot was controlled using wizard of OZ (without telling the players that game is controlled in this way).

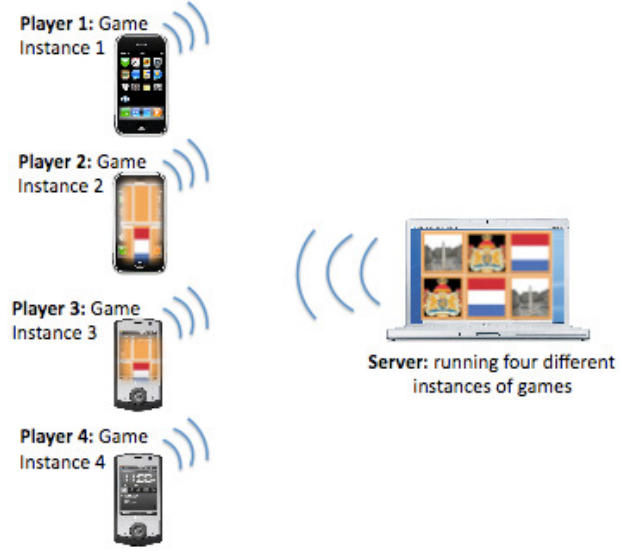

Figure 5.Game network.

\section{RESULTS AND DISCUSSION}

Overall, Walk 2 Win was well appreciated by the participants and the rules well understood. The rules of the game were purposely designed to be simple and that made playing the game easy. One participant said: "The good thing is that the game is not very quick so I can take my time in playing". It was evident that the players got involved with the game and acquainted with the rules from the following strategy employed 
by one of the teams. They deliberately delayed approaching too close to one of the hotspots. They first tried to hear the sound of other neighboring hotspot activated by their opponents and then they choose the spot they were aiming for. This was exemplified in the following quote: "It was rather funny and good when I heard the Queen's sound from Peter's PDA. I was standing in front of wrong hotspot but the sound helped me to move to the right position. I made my pair and peter lost the pair."

The elderly were excited with the prospect of playing Walk 2 Win, as can be explained by the following conversation: "May be I can bring my grand child here with me and we can play together in a team". In response another participant said: "Why don't you bring your Janneke and then you make your own team and I make mine (with my grand daughter) and we can compete". Moving in the game room from one spot to another was also acknowledged. One participant said, "This game made me move, which is quite something". One player suggested that it would be even more interesting if the game room was more spacious. The elderly were fascinated by the dynamic animations used in the game. The elderly wanted the choice of changing the pairs of cards as they desire and suggested having a pair of cards as two pieces of a motif picture. The game was also well received by the caregiver: "I think such games can make them walk, run and also exercise". After the game play session we asked the participants to rank the elements of the game. The PDA ('technology') was ranked as the most appreciated element, followed by physical movement and lastly the cards and sounds from the game. Walk 2 Win became the highlight of the community center, and an audience quickly developed. The audience gave comments and started cheering or jeering the players. But once the game round finished there was a round of applause from the audience for all the players. It was observed during the game playing round that the elderly developed their own strategies to conquer the game, for example they used cues in the environment to remember the location of particular cards. One player informed us later that he actually associated the flower on the table with the hotspot mapped to the queen. The elderly want to connect to their grand child by playing games with them; they find their grandchildren as a window to look into their own children and to connect with them. Recent game research has also shown this trend [1].

There was more tension and competition amongst players while playing W2W individually and not in teams. Sometime they were complaining that their opponents are breaking the rules by coming too close to them while they are activating a hot spot. In such a situation winning seemed to be very important and for them it was more than just a game. On the other hand there was far less animosity while playing $\mathrm{W} 2 \mathrm{~W}$ in teams. There was more collaboration amongst team members and they cooperated in terms of strategy building to find the correct pair of cards.

There were mixed feeling about choosing some strangers as a game partner. One player said if we do not find the right person for our teams then we prefer to play alone. This is very important as we cannot just team up with anybody or any stranger right away. Some said other way around and told that they can team up with stranger for playing such games and may be such games help them communicate with new persons and could be an ice breaker.

\subsection{Implications for Future Game Design}

From our observations and results the following attributes can be valuable to incorporate in game design for the elderly. Firstly, the game should include a natural tendency of audience building. The game should try to involve as many elderly players as possible. The game can have both active and passive participation, as not every senior citizen can play a certain game, due to a limitation (physical, cognitive) and this should not be a reason for them to miss out on the game playing activity. Secondly, the target for any game for the elderly should be Minimum Rules and Maximum Fun. The elderly are very comfortable with uncomplicated rules and they find simple games to the most exciting. Thirdly, the user experience of the elderly can be enhanced by playing known games with add-on's such as technology, new rules, etc. This can make conventional boring games more fun, interesting and enjoyable. Fourthly, games for the elderly should be unisex in nature. At times, females do not play games such as billiard and it would be unfortunate that they are deprived of any game playing experience. To ensure maximum involvement of the community center, participation of both males and females should be encouraged. Finally, the game environment needs to be customizable. The elderly should have the option of changing the game play according to their own wish, consequently motivating them to play the game. Our study provides new insights into game design for the elderly for the purposes of enhancing social interaction and ensuring fun and enjoyment while they are in the community center.

\section{ACKNOWLEDGMENTS}

We are deeply grateful for the assistance provided to us by the community center. A word of gratitude must go out to all the participants of our design case study.

\section{REFERENCES}

[1] Cheok, A.D., Lee, S., Kodagoda, S., Tat, K.E., \& Thang, L.N. (2005). A social and physical inter-generational computer game for the elderly and children: Age invaders. In Proceedings of the 2005 Ninth IEEE International Symposium on Wearable Computers (ISWC'05)

[2] Drew, B. \& Waters, J. (1986). Video games: Utilization of a novel strategy to improve perceptual motor skills and cognitive functioning in the noninstitutionalised elderly. Cognitive Rehabilitation, vol. 4, 26--34

[3] Goldstein, J., Cajko, L., Oosterbroek, M., Michielsen, M., van Houten, O., \& Salverda, F. (1997). Video games and the elderly. Social Behavior and Personality, 25, 345-352

[4] Griffiths, M. (2005). The therapeutic value of video games. In Raessens, J \& Goldstein, J. (Eds.) Handbook of Computer Games Studies. pp.161-171. Cambridge, MA: The MIT Press

[5] Help The Aged (2007), Social Isolation and Lonileness, Help the Aged Policy Statement 2005, available from http://www.helptheaged.org.uk/NR/rdonlyres/477B96FA69C5-4615-8B23AFED030D05C9/0/osteoporosis_statement_130807.pdf

[6] Ijsselsteijn, W., Nap, H. H., de Kort, Y., and Poels, K. (2007). Digital game design for elderly users. In Proceedings of the 2007 Conference on Future Play, Future Play '07. ACM, New York, NY. 17-22

[7] Ijsselsteijn, W., de Kort, Y.A. W., Westerink, J., de Jager, M., \& Bonants, R (2006). Virtual Fitness: Stimulating Exercise Behavious through Media Technology. Presence: Teleoperators and Virtual Environments vol. 15, 688-698

[8] Nielson Interactive Entertainment. (2005). Video Gamers in Europe-2005. Research Report Prepared for the Interactive Software Federation of Europe (ISFE) 